\title{
ANÁLISIS DEL IMPACTO DE LA TRANSPOSICIÓN DE LA DIRECTIVA BOLKESTEIN EN LA PROFESIÓN DE LOS GUÍAS DE TURISMO EN ESPAÑA.
}

\author{
Abril Sellarés, María ${ }^{1}$ \\ Universitat Autònoma de Barcelona \\ Maria.abril@uab.cat
}

Material original autorizado para su primera publicación en la revista académica REDMARKA. Revista Digital de Marketing Aplicado.

https://doi.org/10.17979/redma.2014.01.013.4867

Recibido: 7 Julio 2014

Aceptado 24 Noviembre 2014

\section{RESUMEN}

Desde que España entró en la Unión Europea en el año 1986, su legislación ha tenido que adaptarse a las Directivas que la misma ha ido creando y que le han sido vinculantes. La transposición se corresponde, jurídicamente hablando, al

\footnotetext{
${ }^{1}$ CV: Nacida en Barcelona. Doctoranda por la Universidad de Barcelona (UB) en la línea de investigación de Didáctica del Patrimonio, Museografía y Archivos. Máster en Gestión Cultural y Postgrado en Interpretación del Patrimonio. Profesora desde el año 90 en materias de turismo por la UB y actualmente por la Universidad Autónoma de Barcelona (UAB): patrimonio cultural, destinos turísticos y guías de turismo. Profesora colaboradora en cursos de preparación formativa de los aspirantes a obtener la habilitación de guía de turismo en Catalunya en las materias patrimoniales e históricas y cursos de Capacitación Profesional así como de Ciclos Formativos de Grado Superior en materia turística en procesos de guía y asistencia turística.
}

\author{
REDMARKA UIMA-Universidad de A Coruña - CIECID \\ Año VII, Número 13, (2014), v I pp. 3-16 \\ http://www.redmarka.org/ \\ ISSN 1852-2300
}


mecanismo de despliegue y aplicación de dichas Directivas por parte de las autoridades nacionales competentes ya que, dando sólo las directrices, requiere de un complemento normativo de los Estados para su efectiva implementación (Aguado y Noguera, 2012). En el año 2006 se promulgaba una nueva Directiva 2006/123/CE, conocida como Directiva Bolkestein, relativa a los servicios en el mercado interior, afectando entre otros sectores, al turístico.

Desde la promulgación de la Constitución, en 1978, y en virtud de su artículo 148.1.18 las Comunidades y Ciudades Autónomas (CCAA) tienen traspasadas las competencias en materia turística, lo que ha implicado que desde los gobiernos de dichas CCAA se hayan llevado a cabo dichas transposiciones, (Casanovas, 2011) afectando con ello a los sectores profesionales que forman parte del sector terciario, donde cabe incluir a los guías de turismo.

El presente estudio pretende conocer cómo se ha gestionado dicha trasposición por parte de las CCAA en la normativa que de manera directa o indirecta afecta al sector profesional de los guías de turismo. La metodología que se ha seguido ha consistido en la recopilación, vaciado y análisis de la legislación de cada una de las CCAA para posteriormente ver cómo han hecho la transposición y en qué situación ha quedado la profesión de guía de turismo. Los resultados de la investigación demuestran que no se han seguido criterios unitarios y que en algunas CCAA se ha producido un vacío legal que deja en la más absoluta indefensión esta profesión, dejando, por otro lado la puerta abierta al intrusismo, el cual ha sido un mal endémico en la misma.

Palabras Clave: Directiva Bolkestein, Comunidades Autónomas, Guías de Turismo

\section{A B S T RACT}

REDMARKA UIMA-Universidad de A Coruña - CIECID

Año VII, Número 13, (2014), v I pp. 3-16 http://www.redmarka.org/

ISSN 1852-2300 
Since Spain joined the European Union in 1986, his legislation has had to adapt to the Directives that it has been created by European Union and that have been binding by Spain. The transposition, legally speaking, is a process where the deployment mechanism and implementation of these Directives by national authorities as giving only the guidelines requires a normative complement to states for effective implementation (Aguado \& Noguera, 2012). In 2006 was promulgated a new Directive 2006/123/EC, known as the Bolkestein, on services in the internal market, affecting among other sectors, the tourism.

According to the article 148.1.18 of the Spanish Constitution, the Autonomous Communities (CCAA) and Autonomous Cities have transferred the responsibility in tourism area, so that they corresponded to perform the transposition of Directive Bolkestein to their legislation, (Casanovas, 2011) affecting them to professional sectors part of the tertiary sector, among which is the tourist guides. The study seeks to examine how it has managed that transposition by the CCAA in the regulations that directly or indirectly affects the professional sector of the tourist guides.

The methodology that was followed involved the collection, the analysis and emptying of the legislation of each Autonomous Comunity. Also to see how has transposed it to them and in what situation has remained the profession of tourist guide. The results of research show that there are no uniform criteria followed and in some regions there has been a loophole left in utter helplessness to this profession, leaving the other side open door to the intrusion which has been an endemic problem in it.

Keywords: Bolkestein Directive, Autonomous Communities, Tourist Guide

\section{INTRODUCCIÓN}

REDMARKA UIMA-Universidad de A Coruña - CIECID

Año VII, Número 13, (2014), v I pp. 3-16 http://www.redmarka.org/

ISSN 1852-2300 
La Directiva 2006/123/CE, del Parlamento Europeo y del Consejo, de 12 de diciembre de 2006, relativa a los servicios en el mercado interior también conocida como Directiva Bolkestein ${ }^{2}$, establece una serie de principios aplicables a las normativas reguladoras del acceso a las actividades de servicios y su ejercicio dentro de la Unión Europa (El Parlamento Europeo y el Consejo de la Unión Europea, 2006). Dichos principios tienen como última finalidad, la remodelación, reducción y simplificación de las trabas injustificadas o desproporcionadas para el ejercicio de dichas actividades, ya sea estableciéndose en un Estado miembro, ya sea acogiéndose a la libre circulación de servicios - temporal u ocasional -, proporcionando un entorno más favorable y transparente a los agentes económicos (Razquín, 2010).

A partir de diciembre del año 2009 el ordenamiento jurídico español tenía la obligación de incluir dicha Directiva, provocando con ello una de las reformas más controvertidas dentro de las profesiones liberales, donde se enmarca la de guía de turismo. (Vicente y Rivero, 2009). Las modificaciones tenían que ajustarse a las disposiciones y principios necesarios para garantizar el libre acceso y el ejercicio de las actividades de servicios. En el caso que nos ocupa, se trataba de garantizar el ejercicio de las visitas guiadas realizadas en territorio español por aquellos prestadores establecidos en España o en cualquier otro Estado miembro de la Unión Europea.

En este estudio se pretende conocer cómo han transpuesto la Directiva Bolkestein las diecisiete CCAA en el sector turístico, teniendo presente que las dos ciudades autónomas nunca han legislado sobre dicho tema y que por tanto atienden a la normativa estatal. De modo que, no existiendo una norma común para todo el Estado sobre la regulación de la actividad del guía de turismo es evidente que no hay nada aplicar a dichas ciudades autónomas. Ello ha

\footnotetext{
${ }^{2}$ Frits Bolkestein, fue el comisario europeo para el mercado interno durante la presidencia de Romano Prodi, propuso y defendió esta directiva, a la que, por simplificar, se la suele llamar por el nombre de su creador.
}

REDMARKA UIMA-Universidad de A Coruña - CIECID

Año VII, Número 13, (2014), v I pp. 3-16 http://www.redmarka.org/

ISSN 1852-2300 
motivado que en este estudio sólo se puedan estudiar los casos referidos a las diecisiete CCAA.

Para cumplir con los objetivos del trabajo se optó por una metodología consistente en la búsqueda, lectura y análisis sistematizado de múltiples y variadas publicaciones (artículos, comunicaciones, revistas, entre otros) así como de las normativas correspondientes que afectan directa o indirectamente a la figura profesional de los guías de turismo, es decir, leyes de turismo de modo indirecto y decretos - ley, decretos, reglamentos y órdenes específicamente, y por tanto, directamente, afectando a la actividad del guía. Posteriormente se hizo un vaciado y análisis comparativo de las normas turísticas antes de la Directiva para intentar esclarecer y dilucidar aquellas ideas esenciales sobre la transposición y el estado actual. Dicho vaciado se efectuó a lo largo del año 2013, de modo que sólo se contempla la legislación autonómica hasta el período anterior, es decir el año 2012.

Los resultados obtenidos después del vaciado, análisis y comparación nos demuestran, entre otras cosas, que las CCAA, al igual que habían hecho con sus regulaciones anteriores a la Directiva, siguen criterios propios, sin existir un acuerdo que ayude a uniformar o cuánto mínimo a esclarecer la situación de la actividad profesional objeto de este estudio, dejando a la profesión en un marco no sólo de dudosa legalidad, sino en algunos casos, de legalidad inexistente.

\section{REVISIÓN DE LA LITERATURA}

La aplicación y transposición de esta normativa en el sector turístico español y concretamente a la normativa que afecta a los guías de turismo no ha sido ni fácil ni plácida. Dichas acciones se han producido a dos niveles: el primer nivel referido a la propia normativa estatal y autonómica y el segundo referido a la formación que específicamente necesita el guía de turismo para ejercer correctamente dicha profesión.

REDMARKA UIMA-Universidad de A Coruña - CIECID

Año VII, Número 13, (2014), v I pp. 3-16

http://www.redmarka.org/

ISSN 1852-2300 
Respecto a la normativa, ésta a su vez se divide también en dos niveles: el primero ha tenido unos efectos a escala estatal general donde el Real Decreto 39/2010 de 15 de enero, deroga diversas normas estatales sobre el acceso a las actividades turísticas así como su ejercicio siendo la principal el Decreto 231/1965 de 14 de enero, aún vigente en aquel entonces, por el que se aprobaba el Estatuto ordenador de las Empresas y de las Actividades Turísticas Privadas, en las que se encontraba inmersa la actividad del guía de turismo en sus distintas variantes (Ministerio de Indústria, Turismo y Comercio, 2010). Mientras que el segundo se ha referido al ámbito autonómico. Considerando el hecho de que las CCAA tienen traspasadas las competencias en materia de turismo desde el año 78 y el desenvolvimiento normativo que han realizado según desarrollaban las competencias, ha conducido a una interpretación e implementación variada, lo que ha conllevado un cierto caos en la profesión del guía de turismo después de la Directiva. Así Marcos (2011) señala como, las competencias de las diecisiete CCAA en el territorio que le es propio, han dado lugar a diecisiete formas de interpretar e implementar el proceso de adaptación de las normas provenientes de la CE. A parte quedan las Ciudades Autónomas que no han desarrollado leyes turísticas y por tanto, han quedado subsumidas a la legislación estatal.

Respecto a la formación, el reconocimiento de la misma en la Unión Europea se lleva a cabo a través de las Directivas 89/48/CEE y 92/51/CEE, del "sistema general" de reconocimiento de títulos de enseñanza superior o del reconocimiento de estudios postsecundarios de una duración mínima de un año. Según el nivel de calificación que marca el Estado miembro de acogida, se aplicará la Directiva 89 o la Directiva 92, pero es importante saber que dichas Directivas no crean automáticamente un sistema de equivalencia entre títulos a escala europea. Su principio básico es que el Estado miembro de acogida debe dejar que un nacional comunitario ejerza una profesión en su territorio, aunque no tenga el título nacional requerido, puesto que se le exige el 
título dado por otro Estado miembro para acceder y ejercer dicha profesión. Sólo en el caso de que hayan diferencias sustanciales entre la formación recibida por el profesional y la exigencia del Estado miembro de acogida, éste puede imponer al profesional una medida compensatoria, la cual debe ser entendida, en el caso del guía de turismo como o un período de prácticas o una prueba de aptitud.

Para el objetivo de nuestro estudio y en base a dicha Directiva, lo primero que se observa es que no se especifica a qué profesionales afecta directamente y que la figura del guía de turismo, por ser una profesión liberal, hay que considerar que entra a formar parte de lo que contempla el articulado y concretamente los art. $2.1 \mathrm{y}$ art. 4 .

El art. 2.1 señala el ámbito de aplicación: "La presente Directiva se aplicará a los servicios prestados por prestadores establecidos en un Estado miembro", mientras que el art 4 , define lo que se entiende por servicio, por prestador, por destinatario, por estado miembro de establecimiento, por establecimiento, por estado miembro de destino y por profesión regulada, entre otras variables.

Si de manera deductiva aplicamos ambos artículos al mundo de los guías tenemos que los guías de turismo han de tener unos ítems comunes:

1. Tienen que estar habilitados en un Estado de la Unión Europea.

2. En el caso que quieran ejercer dicha actividad dentro de un espacio declarado patrimonial, estarán obligados a hacer una comunicación previa a la administración correspondiente, la cual determinará qué medidas adoptar para dar el permiso o habilitación correspondiente.

3. Si ejercen su actividad en espacios abiertos y no declarados patrimoniales podrán hacerlo sin necesidad de ningún permiso administrativo.

REDMARKA UIMA-Universidad de A Coruña - CIECID

Año VII, Número 13, (2014), v I pp. 3-16 http://www.redmarka.org/

ISSN 1852-2300 
Autores como Vicente y Casanovas señalan que es justo, en este marco, donde la Directiva puede afectar de manera negativa a la profesión liberal autónoma de los guías de turismo e intérpretes. Sin olvidar, por otro lado, que esta Directiva ha tenido detractores constantes en el sector servicios y muy concretamente en el sector turístico.

A su vez, hay que entender que la afectación normativa puede conllevar una incorporación masiva de trabajadores autónomos que vengan a prestar sus servicios desde otros Estados miembros. Éstos desconociendo la realidad social, económica y política pueden llegar a ejercer su actividad de guiaje turístico con un bajo nivel de conocimientos así como un poco entendimiento con el residente y con los propios profesionales del sector que se sienten apartados (Vicente y Rivero, 2009) (Arcarons, Casanovas, \& Giménez, 2007).

Por otro lado hay que tener en cuenta que la profesión del guía de turismo es una de las que más padece de intrusismo y que a pesar de que haya decretos, reglamentos y órdenes que su actividad, su control pasa por ser sumamente difícil, ergo la casuística normativa tampoco ha ayudado a que se tengan claros los límites de la profesión ni a cómo combatir dicho intrusismo (Picazo, 2011). De modo que con la Directiva la problemática del intrusismo se mantiene y muy probablemente aumente por las interpretaciones que los prestadores puedan hacer sobre la aplicación e implementación aumente.

Por su parte, Casanovas (2011) señala como ha tenido que variar el marco jurídico de la actividad de los guías de turismoy la confusión de aplicación desde las diferentes administraciones autonómicas, dado que las propias administraciones han desarrollado normativas diferentes y en ocasiones hasta dispares sobre esta profesión.

\section{SITUACIÓN DE LA TRANSPOSICIÓN EN LAS CCAA: ANÁLISIS Y RESULTADOS}

REDMARKA UIMA-Universidad de A Coruña - CIECID

Año VII, Número 13, (2014), v I pp. 3-16

http://www.redmarka.org/

ISSN 1852-2300 
A través del vaciado normativo que se ha realizado y del análisis comparativo deductivo se han alcanzado los objetivos que se pretendían con este estudio se ha podido configurar un mapa, un tanto desolador de la evolución real a nivel legal de la actividad turístico guiada en España. Desde la Constitución de 1978, las CCAA han legislado de manera irregular e independiente el sector turístico dentro de su ámbito de aplicación. Ello ha dado lugar a una casuística, interpretación e implementación coincidente en algunos casos y en otros, totalmente dispares.

Para poder trabajar los datos obtenidos se han utilizado dos variables que ayudan a sistematizar el estudio:

Primera variable: Las Normas relacionadas con los Guías de Turismo en las CCAA, antes de la Directiva Bolkestein

$\square$ Segunda variable: la transposición de la Directiva de Bolkestein en cada CCAA y las acciones generadas.

Antes de la Directiva Bolkestein. De las 17 CCAA hemos encontrado que 15, es decir un $88 \%$ de las mismas, han promulgado normas que afectan en alguna parte de su articulado a la figura profesional del guía de turismo, y más concretamente a la regulación de su actividad. Por tanto podríamos decir que se sientan los criterios base pero no desarrollan la figura como tal ya que para ello se necesita de normas de rango inferior que permiten el desarrollo de la norma de rango superior. Se trata de las Leyes que versan sobre la ordenación turística del territorio o leyes de turismo.

Las dos CCAA que no habían promulgado Leyes turísticas fueron La Rioja y Navarra. En el caso de la CA de La Rioja se promulgó una Ley la de 5/1990, de 29 de junio, que hacía referencia a temes de: inspección, infracciones y sanciones en materia de Turismo, pero, en ninguna parte de su articulado se regulaban ni las actividades ni las profesiones. Por su parte la Comunidad Foral

REDMARKA UIMA-Universidad de A Coruña - CIECID

Año VII, Número 13, (2014), v I pp. 3-16 http://www.redmarka.org/

ISSN 1852-2300 
de Navarra promulgó la Ley Foral 14/1997, de 17 de noviembre, de disciplina turística en Navarra, la cual limitada como su denominación indica a los aspectos de disciplina y régimen sancionador deja de regular el ámbito de actuación y marco de las actividades así como las profesiones de la susodicha disciplina turística. El hecho de no haber promulgado leyes turísticas no significa que no hayan regulado específicamente las actividades del sector turístico.

Lo que nos llevaba al segundo ítem a valorar, qué CCAA hacían regulaciones de modo directo y cuáles lo hacían de modo indirecto. Respecto a aquellas CCAA que de manera directa a través de decretos, reglamentos, órdenes, o disposiciones han regulado la actividad y profesión del guía de turismo hemos obtenido los siguientes resultados. 14 de las CCAA han regulado directamente, lo que significa un $82,35 \%$ del total de las 15 CCAA, y 3 que lo han hecho de manera indirecta: Galicia, País Vasco y Asturias, lo que significa un total de $17,64 \%$ del total.

Este 17,64\% asimilaba los principios rectores de sus leyes base, pero no hacía aplicativas las mismas por no tener leyes posteriores que permitiesen su utilización, quedando como único recurso el llamar a una ley estatal como era la Orden de 31 de enero de 1964 que promulgaba el Reglamento para el ejercicio de actividades turísticas-informativas privadas la cual había sido creada por el Ministerio de Información y Turismo. El uso de dicha Orden fue posible, en tanto en cuanto, España estaba fuera de la Comunidad Europea, pero a partir del año 86, sus normativas tuvieron que irse adaptado a las ya existentes en la Unión Europea, hecho que provoca una contradicción de esta Orden con la Directiva de la Unión Europea sobre la libre prestación de servicios. De modo que, el Reino de España el 22 de marzo de 1994, es condenado por la CEE, por no cumplir la libre prestación de servicios y la consecuencia inmediata es la derogación de dicha Orden. 
En consecuencia, para aquellas CCAA que mantenían la Orden de 31 de enero de 1964 en su cuerpo legislativo, se evidenció la falta de normativa existente y lo que es más grave aún, la no voluntad de llevar a cabo una nueva normativa, dejando entre 1994 y 2006 un vacío legal importante. Mientras para aquellas CCAA que habían regulado y reglamentado la profesión del guía de turismo, más del 82\% se iniciaban períodos de cambios. La Sentencia de 1994 que obligaba a España a cumplir la libre prestación de servicios, provocaba una modificación en las normativas que contradecían a dicha sentencia. Dicha modificación fue avalada, con una cierta celeridad por el $100 \%$ de todas las CCAA afectadas. En cambio la Directiva Bolkestein aprobada en el año 2006 por parte de la Unión Europea y ratificada por España, mostraría líneas de actuación diversas entre los legisladores de las distintas regiones autónomas.

Después de la Directiva Bolkestein. Desde que se produce la Directiva hasta su aplicación efectiva las CCAA tenían un plazo de tiempo para adaptar sus normativas turísticas a la misma, y si bien el panorama normativa ha cambiado la casuística se mantiene. Además no todas las CCAA han adaptado su legislación a la Directiva, lo que significa que siendo contrario a una norma de rango superior, queda automáticamente invalidada/derogada la norma de rango inferior en aquellos apartados que contradigan sus disposiciones. Esto ha provocado un nuevo vacío legal al derogar las leyes, reglamentos $u$ órdenes que eran contrarias a la misma en aquellas CCAA que de un modo directo o indirecto habían regulado la actividad de los guías de turismo. El panorama legislativo actual muestra una situación un tanto confusa a finales del año 2012 de las 17 CCAA:

$\square 15$ de las CCAA han promulgado o modificado las Leyes de Turismo, 2 CCAA Navarra, y Valencia aún no han actuado ni con modificaciones ni con nuevas legislaciones. Recordemos que como tales leyes necesitan 
de reglamentos posteriores, ordenes o decretos para desarrollarlos, hecho que no se ha producido.

$\square$ Respecto a la legislación que reglamenta la actividad de guía, tenemos:

a) 4 CCAA han creado nuevos decretos: Aragón, Extremadura, Galicia, Asturias. Mención especial merece Galicia, ya que antes de la Directiva no había creado un reglamento propio de los guías.

b) 8 CCAA han modificado sus reglamentos, órdenes y/o decretos.

c) 5 CCAA que hasta la fecha no han actuado ni modificando ni creando nuevos reglamentos. Lo cual provoca no sólo un desajuste sino un vacío legal.

\section{CONCLUSIONES}

La peculiaridad de las CCAA en el territorio español y el hecho de que tienen traspasadas las competencias en materia de turismo ha producido, desde que iniciaron sus legislaciones propias, una casuística, una interpretación y una implementación de sus leyes en el territorio según las necesidades e idiosincrasia del mismo. La reglamentación del mundo de los guías no ha sido ninguna excepción a este hecho, lo que ha creado confusiones y en algunos casos desconcierto a la hora de acometer, acotar y conocer la realidad de esta profesión.

La Directiva Bolkestein ha enmarcado una serie de principios que tienen como última finalidad la remodelación, reducción y simplificación del ejercicio de las actividades libre profesionales. Pero se ha podido comprobar que en lugar de ayudar, en el caso español, la situación de confusión y de vacío legal se sigue manteniendo.

Así tenemos que con la entrada de España a la Unión Europea y lo que ello conlleva, entre otras cosas, la transposición de sus Directivas se podría haber planteado la unificación de criterios, tal y como vieron en su momento autores

REDMARKA UIMA-Universidad de A Coruña - CIECID

Año VII, Número 13, (2014), v I pp. 3-16 http://www.redmarka.org/

ISSN 1852-2300 
como Casanovas (2011) o Picazo (2011). La realidad es que dicha transposición pone en evidencia el escaso conocimiento que existe sobre la profesión del guía de turismo provocando situaciones tan perplejas como: que se lleguen a crear vacíos legales en CCAA donde se había regulado la profesión o que la permisividad al intrusismo se ponga de manifiesto sin traba alguna, al no quedar acotación sobre el marco de la actividad y sobre el carácter profesional del guía así como de su mundo laboral. Consecuencia de ello, es la alarma social entre los miembros de la profesión que sin con anterioridad a la Directiva Bolkestein ya les era difícil mantener criterios saludables para la profesión, desde la transposición de la misma les está costando que se pueda alcanzar un criterio claro, objetivo y razonable de cómo afrontar una realidad social. Han pasado de sentirse mínimamente amparados por la ley o el olvido más absoluto y el desamparo más manifiesto.

\section{REFERENCIAS}

Aguado, V. y Noguera, B. (2012). El impacto de la Directiva de servicios en el turismo en las administraciones públicas, aspectos generales y sectoriales. Atelier.

Aragón Canovas, F.J. [et.al]. (2004). Turismo y Derecho. Salamanca: Aquilafuente.

Arcarons, R., Casanovas, O., y Giménez, A. (2007). Manual de Derecho Laboral Turístico. Madrid: Síntesis.

B.O.E. (1952). Boletín Oficial del Estado. 214,3576.

Blasco, A. (2006). Tipologías turísticas. Girona: Xarxa d'Escoles de Turisme.

Casanovas, O. (30 de mayo de 2011). El Derecho Turístico. (C. info, Entrevistador)

REDMARKA UIMA-Universidad de A Coruña - CIECID

Año VII, Número 13, (2014), v I pp. 3-16

http://www.redmarka.org/

ISSN 1852-2300 
Comisión de las Comunidades Europeas. (2000). Informe de la Comisión al Consejo y al Parlamento Europeo sobre la aplicación de la Directiva 92/51 CEE dirigido de conformidad con el artículo 18 de la Directiva 92/51/CEE. Bruselas: COM.

Marcos, J. (22-03-2011). La Directiva Bolkenstein y el turismo en España: el estado de la cuestion. Hosteltur. Recuperado el 22 de 02 de 2012, de Hosteltur: http://www.hosteltur.com/131500_directiva-bolkestein-turismoespana-estado-cuestion.html

Ministerio de Indústria, Turismo y Comercio. (04-02-2010). Real Decreto 39/2010 de 15 de enero, por el que se derogan diversas normas estatales sobre acceso a actividades turísticas y su ejercicio. Boletín Oficial del Estado. Madrid, Madrid, España: BOE.

Picazo, C. (2011). Procesos de guía y asistencia turística. Madrid: Síntesis.

Razquín, J. (2010). El impacto de la Directiva de Servicios en el procedimiento administrativo: autorización, declaración responsable y comunicación. Revista Jurídica de Navarra, 85-136.

Vicente, D.-J., y Rivero, R. (2009). Impacto de la transposición de la Directiva de Servicios en Castilla y León. Valladolid: Consejo Económico social de Castilla-León.

REDMARKA UIMA-Universidad de A Coruña - CIECID

Año VII, Número 13, (2014), v I pp. 3-16 http://www.redmarka.org/

ISSN 1852-2300 